Combined NC-AFM and DFT study of the adsorption geometry of trimesic acid on rutile $\mathrm{TiO}_{2}(110)$

This article has been downloaded from IOPscience. Please scroll down to see the full text article.

2010 J. Phys.: Condens. Matter 22345008

(http://iopscience.iop.org/0953-8984/22/34/345008)

View the table of contents for this issue, or go to the journal homepage for more

Download details:

IP Address: 134.93.197.106

The article was downloaded on 12/08/2010 at 15:58

Please note that terms and conditions apply. 


\title{
Combined NC-AFM and DFT study of the adsorption geometry of trimesic acid on rutile $\mathrm{TiO}_{2}(110)$
}

\author{
Andreas Greuling, Philipp Rahe ${ }^{1}$, Marcin Kaczmarski, \\ Angelika Kühnle ${ }^{1}$ and Michael Rohlfing
}

Fachbereich Physik, Universität Osnabrück, Barbarastrasse 7, Osnabrück 49069, Germany

Received 19 May 2010, in final form 30 June 2010

Published 12 August 2010

Online at stacks.iop.org/JPhysCM/22/345008

\begin{abstract}
The adsorption behavior of trimesic acid (TMA) on rutile $\mathrm{TiO}_{2}(110)$ is studied by means of non-contact atomic force microscopy (NC-AFM) and density-functional theory (DFT). Upon low-coverage adsorption at room temperature, NC-AFM imaging reveals individual molecules, centered above the surface titanium rows. Based on the NC-AFM results alone it is difficult to deduce whether the molecules are lying flat or standing upright on the surface. To elucidate the detailed adsorption geometry, we perform DFT calculations, considering a large number of different adsorption positions. Our DFT calculations suggest that single TMA molecules adsorb with the benzene ring parallel to the surface plane. In this configuration, two carboxylic groups can anchor to the surface in a bidentate fashion with the oxygen atoms binding to surface titanium atoms while the hydrogen atoms approach oxygen atoms within the bridging oxygen rows. The most favorable adsorption position is obtained in the presence of a hydroxyl defect, allowing for additional binding of the third carboxylic group.

(Some figures in this article are in colour only in the electronic version)
\end{abstract}

\section{Introduction}

The binding of organic molecules onto surfaces is of great interest in fields such as catalysis [1], surface functionalization and corrosion protection [2] as well as bio-sensing [3]. Moreover, for employing molecular self-assembly [4] in future molecular electronic devices [5], a detailed understanding of molecule-substrate interaction is required.

Consequently, the adsorption of molecules on surfaces has attracted considerable attention in the last few decades and has been investigated both experimentally $[6,7]$ as well as theoretically $[8,9]$. So far, however, the vast majority of studies have been carried out using metallic rather than oxide surfaces. This is largely due to the fact that oxide surfaces often constitute a great challenge since they combine localized and delocalized electronic states and frequently show weak interaction with adsorbates. Also from an experimental point of view, studying oxide surfaces can be very demanding as many surface science techniques require conductive substrates

\footnotetext{
1 Present address: Institut für Physikalische Chemie, Johannes GutenbergUniversität Mainz, Jakob-Welder-Weg 11, 55099 Mainz, Germany.
}

and are, therefore, not suited for studying insulating oxide materials.

Recently, molecular adsorption studies have been extended to oxide surfaces, with rutile $\mathrm{TiO}_{2}(110)$ being a very prominent model surface due to its manifold applications in a wide range of different fields [10]. In particular, the adsorption of organic molecules containing carboxylic groups has been investigated both theoretically based on density-functional theory (DFT) [11-14] and experimentally using scanning tunneling as well as atomic force microscopy [15-20]. Being a versatile molecular linker, terephthalic acid (TPA) has been studied recently on $\mathrm{TiO}_{2}(110)$, revealing a transition from flatlying molecules at low coverage to upright-standing molecules at high coverage [17, 18].

Trimesic acid (TMA), inhibiting one additional carboxylic moiety compared to TPA, has been discussed as a molecular building block for highly ordered structures on conducting surfaces such as HOPG, $\mathrm{Cu}, \mathrm{Au}$ and $\mathrm{Ag}$ [19, 21-25]. On these surfaces, the flat-lying TMA molecules form characteristic 'flower' and 'chicken wire' structures stabilized by hydrogen bonds between the carboxylic acid groups. In contrast, it is generally assumed for carboxylic acid groups on $\mathrm{TiO}_{2}(110)$ 


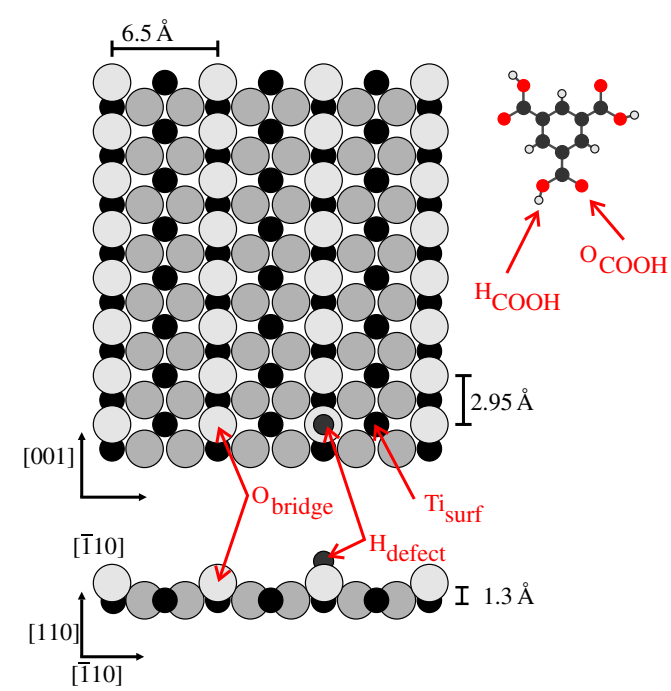

Figure 1. Model of the $\mathrm{TiO}_{2}(110)$ surface. Black, bright gray and dark gray circles represent the $\mathrm{Ti}$, bridging oxygen and in-plane oxygen atoms, respectively. A model of a TMA molecule is drawn to scale.

to dissociate at room temperature, giving carboxylate and hydrogen, although theoretical calculations have yielded rather similar binding energies for the protonated and deprotonated form $[11,26,27]$. For small molecules, the carboxylate adsorbs on a pair of 5-fold coordinated $\mathrm{Ti}$ atoms in a bridge form with the $\mathrm{O}-\mathrm{C}-\mathrm{O}$ plane aligned along the [001] crystallographic direction. So far, larger molecules are assumed to follow this trend $[18,28]$.

Here, we study the adsorption of trimesic acid $\left(\mathrm{C}_{6} \mathrm{H}_{3}(\mathrm{COOH})_{3}\right.$, TMA $)$ on $\mathrm{TiO}_{2}(110)$, to gain insights into the detailed adsorption geometry. The surface structure of $\mathrm{TiO}_{2}(110)$ and a model of a TMA molecule drawn to scale are presented in figure 1.

Upon low-coverage deposition at room temperature under ultra-high vacuum (UHV) conditions, our non-contact atomic force microscopy (NC-AFM) experiments reveal individual molecules being centered above the surface titanium rows. From the NC-AFM images alone it remains, however, difficult to deduce the molecular binding configuration. To tackle the question of the detailed binding situation, we complement the experimental results with DFT calculations. Using the SIESTA [29, 30] code, we explore the binding behavior of a large number of different adsorption configurations, including deprotonated TMA. Besides the stoichiometric surface, we also consider hydroxyl defects, which are known to considerably alter the binding situation of organic molecules [31]. In the absence of surface defects, we find TMA adsorbing in a flat-lying geometry with two carboxyl groups binding in a bidentate fashion to the surface. The oxygen atoms of the two carboxylic groups $\left(\mathrm{O}_{\mathrm{COOH}}\right)$ bind towards the surface titanium atoms $\left(\mathrm{Ti}_{\text {surf }}\right)$ while the hydrogen atoms $\left(\mathrm{H}_{\mathrm{COOH}}\right)$ anchor to the bridging oxygen atoms $\left(\mathrm{O}_{\text {bridge }}\right)$, forming a hydrogen bond. This adsorption mechanism yields a binding energy of $2.16 \mathrm{eV}$ (see below). The binding energy is even higher (by $0.2 \mathrm{eV}$ ) if adsorption occurs on top of a hydroxyl defect of the $\mathrm{TiO}_{2}$ surface. In this case, the third carboxylic group can also form a bond towards the surface by bending its oxygen atom $\left(\mathrm{O}_{\mathrm{COOH}}\right)$ towards the hydrogen atom at the surface $\left(\mathrm{H}_{\text {defect }}\right)$. As a consequence of enhanced binding to surface defects, diffusion of the TMA molecules would be suppressed. These findings suggest that surface hydroxyl defects, which are inevitably obtained when preparing $\mathrm{TiO}_{2}(110)$ in UHV, play a central role for anchoring of the molecules towards the surface.

The paper is organized as follows. Section 2 describes the experimental techniques and discusses the measured AFM data of TMA on $\mathrm{TiO}_{2}$. In section 3 we discuss the theoretical approach and investigate the resulting structural and energetic data for the adsorption of TMA. A short summary concludes the paper.

\section{Non-contact atomic force microscopy}

NC-AFM measurements were performed using a VT AFM 25 from Omicron (Taunusstein, Germany) under UHV conditions [31]. In NC-AFM, a sharp tip mounted to the end of an oscillating cantilever is brought near the surface, sensing forces acting between the foremost tip atoms and surface species. These forces shift the current oscillation frequency $f$ relative to the resonance frequency $f_{0}$ of the free oscillating cantilever. The frequency shift $\Delta f=f-f_{0}$, referred to as detuning, is the main measuring signal and is sampled in a rectangular array of 500 pixel $\times 500$ pixels. Additionally, the signal may be fed into a distance regulation, keeping $\Delta f$ at a certain set point. In this constant-detuning mode, the main signal is the topography channel. The pictures shown in this work are taken in an intermediate constant-height/constantdetuning mode for tip stability reasons [32]. The $\mathrm{TiO}_{2}$ samples were of the highest available quality from MTI (Richmond, USA). The (110) surfaces were prepared by several cycles of $\mathrm{Ar}^{+}$sputtering at $1 \mathrm{keV}$ and annealing at $\sim 1100 \mathrm{~K}$, each step lasting for $15 \mathrm{~min}$. Both, molecule deposition and NCAFM measurements were performed with the sample held at room temperature. The TMA molecules (purity of 97\%) were bought from Sigma-Aldrich. They were sublimated from a glass crucible held at a temperature of around $\sim 410 \mathrm{~K}$. The corresponding rate of $\sim 2.9 \mathrm{ML} \mathrm{h}^{-1}$ was calibrated using a quartz crystal microbalance ${ }^{2}$.

For imaging $\mathrm{TiO}_{2}(110)$ using NC-AFM, different contrast mechanisms have been reported [33-36]. In all imaging modes, rows along the [001] crystallographic direction are easily resolved. The assignment to in-plane titanium or protruding oxygen rows, however, requires identification of the surface defects: vacancies as well as hydroxyls are located on the bridging oxygen rows exclusively.

The three most prominent imaging modes have been explained with a simple model based on electrostatic interaction: in the so-called 'hole-mode' imaging, bridging oxygen rows are imaged bright with the defects appearing as dark depressions along the bright oxygen rows. This mode was explained by a positive tip termination, yielding the largest

2 For our setup, one monolayer (1 ML) of TMA on $\mathrm{TiO}_{2}(110)$ is reached after sublimating the TMA at $411 \mathrm{~K}$ for $(1060 \pm 70) \mathrm{s}$. 


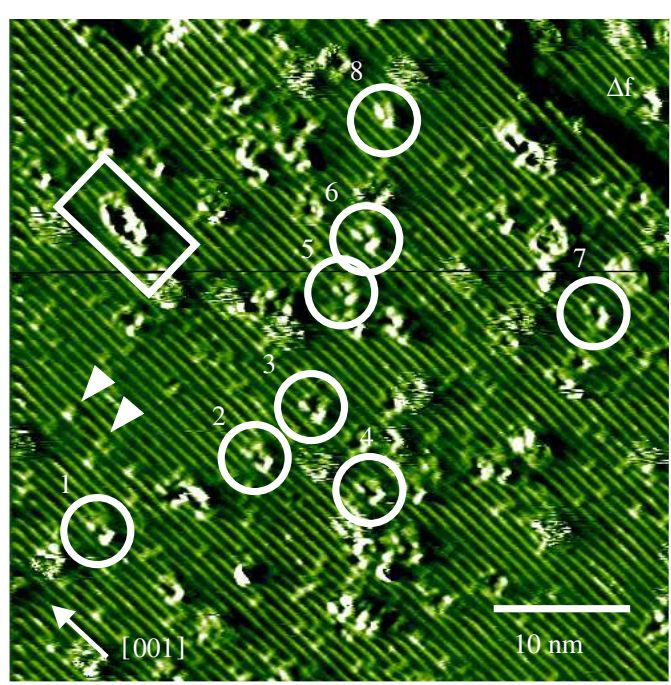

Figure 2. $\mathrm{TiO}_{2}(110)$ with adsorbed TMA molecules, sublimated for 5 min at $407 \mathrm{~K}$ (raw data detuning image, the fast scan direction is to the right, the slow scan direction is down). Several molecules are marked by a white circle, one larger molecular cluster is marked by a white rectangle. Furthermore, we can identify the bright rows as in-plane titanium rows due to the presence of bright defects in between them; two are marked by small triangles. This imaging mode has been referred to as the 'protrusion mode', which has been explained by a negative tip termination [33]

interaction with the bridging oxygen rows $[33,34]$. In the socalled 'protrusion-mode' imaging, in-plane titanium rows are imaged bright with the defects appearing as bright linkers in between these rows. This mode was explained by a negative tip termination, yielding the largest interaction with the titanium atoms [33, 34]. In the so-called 'neutral' mode, the bridging oxygen rows are imaged bright, with the defects appearing as bright protrusions on top of these rows $[34,35]$.

Recently, the imaging of the in-plane titanium atoms additionally to the bridging oxygen rows has also been reported [35], as well as the imaging of the in-plane oxygen rows, performing experiments at $80 \mathrm{~K}$ [36].

Figure 2 presents the $\mathrm{TiO}_{2}(110)$ surface after sublimating TMA for $5 \mathrm{~min}$ with the glass crucible held at $407 \mathrm{~K}$. Concerning the substrate, bright rows along the [001] crystallographic direction are visible. These rows are interconnected by bright 'linkers', two of which are marked by small white triangles. We attribute these linkers to hydroxyl defects and, consequently, identify the bright rows as in-plane titanium rows (the so-called protrusion mode, which has been explained by a negative tip termination $[33,34])$.

The TMA molecules appear to be immobile and are imaged as rather large protrusions with the molecular center being positioned above a surface titanium row. The position along the [001] crystallographic direction cannot be deduced from the images as atomic resolution along this direction was not obtained. Some of the molecules appear to possess a three-fold structure: two bright stripes are aligned along the [001] crystallographic direction and a third stripe along the [110]. This structure is visible in most of the molecules present, sometimes less pronounced. Note that the dark center may be an imaging artifact: as an intermediate constantheight/constant-detuning mode was used, the tip may penetrate into the repulsive regime [32].

Considering the molecular structure, this three-fold structure might be explained by the three carboxylic groups attached to the benzene core of a flat-lying TMA molecule. However, as we do not know the exact tip geometry we cannot exclude image artifacts from, e.g., a multiple tip. Thus, based on the NC-AFM images alone, the detailed adsorption configuration cannot be deduced.

Very rarely, diffusion events were observed. Interestingly, when a molecule diffuses, the motion is very fast, actually much too fast to experimentally determine the corresponding hopping rate. The fast diffusion contradicts the small number of diffusion events observed. This discrepancy can be resolved by considering different adsorption positions, including stable anchoring sites such as surface defect positions.

\section{Density-functional theory}

To complement the NC-AFM results and to elucidate the detailed adsorption geometry, we performed DFT calculations using the SIESTA package. We used the local density approximation (LDA) with a Ceperley Alder exchangecorrelation potential [37], a mesh cutoff of 130 Ryd and a double zeta polarized (DZP) basis set [30]. Gradientcorrected exchange-correlation functionals sometimes yield qualitatively wrong results for weakly interacting adsorption systems (like, e.g., PTCDA on $\operatorname{Ag}(111)$, which does not bind in GGA at all), for which LDA has been shown to perform in good agreement with more elaborate exchange-correlation total-energy methods [38-40]. We therefore employ LDA to describe the adsorption mechanisms, although LDA sometimes tends to overbind. Our results for the adsorption energies may thus be understood as upper limits. The most extended orbitals are truncated at a radius of $3.75,2.74,3.57$ and $3.45 \AA$ for $\mathrm{Ti}$, $\mathrm{O}, \mathrm{C}$ and $\mathrm{H}$, respectively. For titanium we considered the $3 \mathrm{~s}, 3 \mathrm{p}$ and $3 \mathrm{~d}$ orbitals as valence orbitals, while all other orbitals were taken as core states and were replaced by norm-conserving nonlocal Troullier-Martins pseudopotentials [41]. The cutoff radii were chosen as $1.0(1.9,1.1)$ bohr for $\mathrm{Ti} 3 \mathrm{~s}(3 \mathrm{p}, 3 \mathrm{~d})$, 1.15 bohr for $\mathrm{O} 2 \mathrm{~s}$ and $2 \mathrm{p}$, and $1.25 \mathrm{bohr}$ for $\mathrm{C} 2 \mathrm{~s}$ and $2 \mathrm{p}$, respectively.

First of all, we relax the bulk structure for rutile $\mathrm{TiO}_{2}$ starting from experimental lattice constants $(a=b=$ $4.5929 \AA, c=2.9591 \AA$ ) [42] and end up with a structure showing a small deviation of less than $1 \%(a=b=4.5839 \AA$, $c=2.9406 \AA$ ). This value of $a$ is then employed for the lateral lattice constant of the surface, in order to achieve mechanical consistency between bulk and surface physics. The surface is first described by a slab of 11 (atomic) layers (slab thickness: $12.2 \AA$ ). We relaxed the outermost nine layers while keeping the two lowest layers at bulk positions. In order to reduce the numerical cost to a reasonable amount, we then only considered the upmost six layers of this slab (12 atoms per $1 \times 1$ surface unit cell) for all the following calculations, fixing the coordinates of the two lowest layers. The relaxation of the systems, consisting of the $\mathrm{TiO}_{2}(110)$ surface and one 


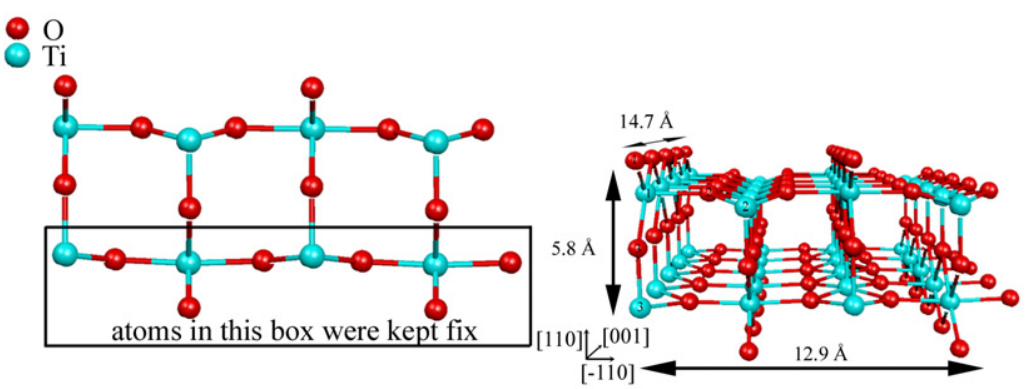

Figure 3. $\mathrm{TiO}_{2}$ (110) slab consisting of six (atomic) layers as used in the DFT calculations.

TMA molecule in various starting configurations, took place in a $5 \times 2$ surface unit cell $(14.7 \AA \times 12.9 \AA)$ of $32 \AA$ height (figure 3 ). This supercell size leads to a sufficiently thick vacuum $(\sim 19 \AA$ for molecules standing upright over the titanium rows and $>20 \AA$ for all other configurations) above the surface, ensuring the absence of significant spurious interaction with the periodically repeated cell. The lateral distance between two TMA molecules in neighboring cells is also large enough to suppress significant direct interactions. This distance is at least $4 \AA$. In most cases, however, it is considerably larger, as the molecules tend to bend.

In all cases the combined system of one TMA molecule on top of the six-layer $\mathrm{TiO}_{2}$ slab was relaxed until the forces acting on each atom were smaller than $0.01 \mathrm{eV} \AA^{-1}$. We then evaluated two different definitions of the binding energy. We define $E_{\text {int }}$ as the energy needed to separate the surface and the molecule without allowing relaxation of surface or molecule afterward and $E_{\mathrm{b}, \mathrm{r}}$ as the energy with allowed relaxation. To calculate the electronic interaction energy $\left(E_{\text {int }}\right)$ we take the total energy of the relaxed TMA + surface system $\left(E_{5}\right)$ and subtract the individual total energies of the isolated TMA $\left(E_{1}\right)$ and of the surface $\left(E_{2}\right)$ with their structures being those of the adsorption configuration. This binding energy measures the change of the total energy due to rearranging the electrons when two objects are brought in contact without changing their internal structures, i.e. taking the internal structures from the relaxed adsorption configuration. The relaxed binding energy $\left(E_{\mathrm{b}, \mathrm{r}}\right)$, on the other hand, is calculated by subtracting the individual total energies of the free molecule $\left(E_{3}\right)$ and the free surface $\left(E_{4}\right)$ in their individually relaxed structures. $E_{\mathrm{b}, \mathrm{r}}$ thus includes the (positive) deformation energy necessary to deform both components (molecule and surface) into the final structures which they take in the adsorption state. The binding energy $E_{\mathrm{b}, \mathrm{r}}$ describes the entire change of the total energy when a gas-phase TMA molecule adsorbs on $\mathrm{TiO}_{2}$ or when an adsorbed molecule desorbs into the gas phase on timescales larger than those of structural relaxation (as, e.g., in calorimetric measurements). $E_{\text {int }}$, on the other hand, is a useful tool for discussing electronic effects, the formation of chemical bonds between molecule and surface, and quick processes on short timescales that are too fast to allow for internal structural deformation, like electronic processes, bond breaking by light, etc. The thermodynamical stability of one configuration with respect to another, as well as the search for the lowest-energy adsorption structure, is controlled by $E_{\mathrm{b}, \mathrm{r}}$.

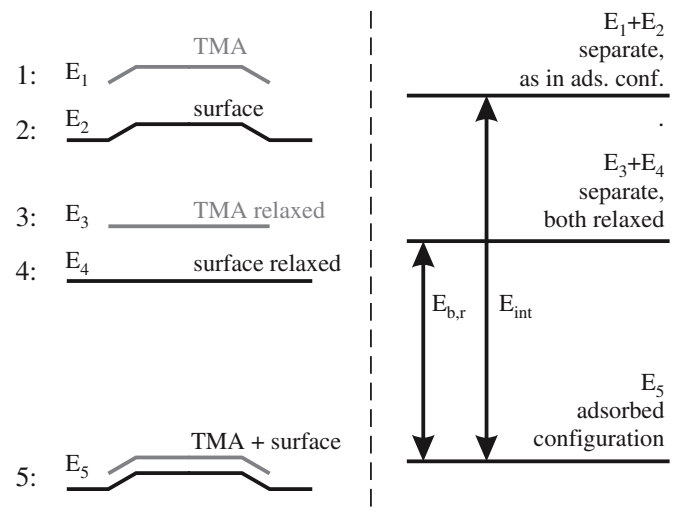

Figure 4. Definition of the various total energies (for the respective configurations), required for the evaluation of the binding energies (see text).

For both types of binding energy we correct for the basisset superposition error $\left(E_{\mathrm{BSSE}}\right)$ by employing the so-called counterpoise (CP)/ghost orbital approach [43, 44]. A ghost orbital can be thought of as an additional basis-set piece (i.e., from a binding partner), which may improve the incomplete basis set of an atom. Finally, this leads to a different (lower) energy, which should be corrected if one wants to compare binding energies. Thus, the two binding energies are defined as follows (see visualization in figure 4):

$$
\begin{aligned}
& E_{\mathrm{int}}=E_{5}-E_{1}-E_{2}-E_{\mathrm{BSSE}} \\
& E_{\mathrm{b}, \mathrm{r}}=E_{5}-E_{3}-E_{4}-E_{\mathrm{BSSE}} .
\end{aligned}
$$

In the following, we define the total energy of the TMA interacting with the ghost orbitals of the surface (geometry as in figure 4 configuration 5) as $E_{1+\text { ghost }}$, and the total energy of the surface interacting with the ghost orbitals of the TMA molecule as $E_{2+\text { ghost }}$. We then obtain the basis-set superposition error as

$$
E_{\mathrm{BSSE}}=\left(E_{1+\text { ghost }}-E_{1}\right)+\left(E_{2+\text { ghost }}-E_{2}\right) .
$$

This only takes into account the BSSE coming from the interaction between the surface and the TMA and not the BSSE 'inside' the TMA (surface) system, which is the error from each atom interacting with the ghost orbitals of all other atoms in the TMA (surface) system. We assume that the BSSE 


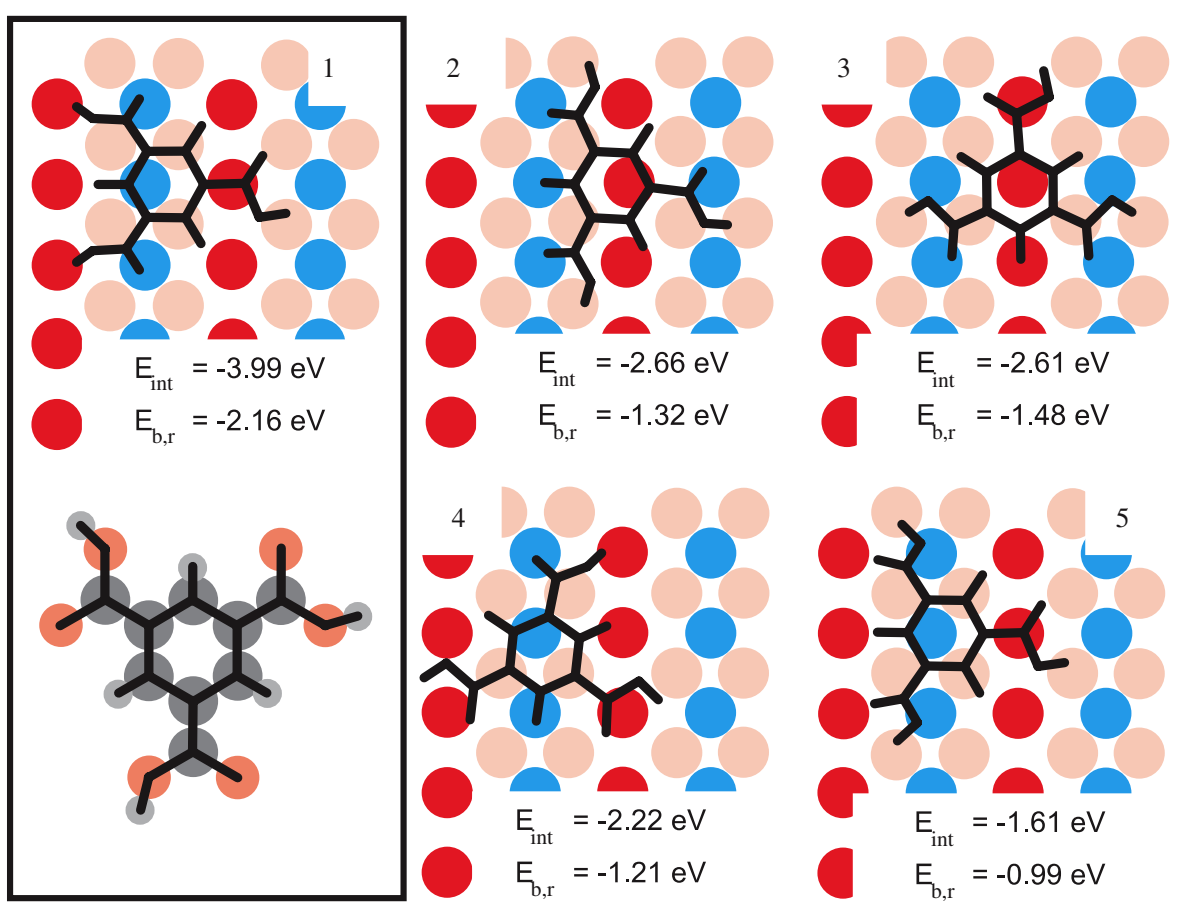

Figure 5. Relaxed configurations of the TMA molecule on the stoichiometric $\mathrm{TiO}_{2}(110)$ surface. Note that the projection of the molecules on the surface plane is shown, resulting in an asymmetric appearance of molecules that adsorb in a tilted configuration. Configuration 1 is the most stable one.

'inside' the unrelaxed TMA (surface) system is the same as for the relaxed TMA (surface), and they thus cancel each other.

Finding the optimum configuration of a rather large system as considered here is a difficult problem. The high computational cost for the calculation of one single configuration and the large number of possible configurations make it difficult to find the global total-energy minimum. In particular, most conventional relaxation algorithms (like, e.g., the Broyden [45] or Conjugate Gradient [46] method) might get trapped in local minima, depending on the starting configuration. Therefore we have considered a large number of realistic starting configurations in a systematic manner. They include both flat-lying as well as upright-standing molecules in deprotonated and intact molecular form, yielding a comprehensive sampling of the configurational space. The starting positions for the flat-lying molecules are either centered over the titanium rows or over the bridging oxygen rows, including different orientations. Molecules in a standing configuration are considered in different orientations centered above a surface titanium row.

Figure 5 shows the five lowest-energy adsorption configurations of TMA on the stoichiometric surface (i.e., without surface defects), obtained after relaxation. For configuration 1 the benzene ring of the TMA molecule is tilted by $6^{\circ}$ with respect to the [001] direction. The angles for configuration $2-5$ are between $5^{\circ}$ and $14^{\circ}$. Configuration 1 gives an adsorption energy $E_{\mathrm{b}, \mathrm{r}}$ of $-2.16 \mathrm{eV}$, which is significantly more stable than all other structures tested. This results from the formation of bonds between two carboxylic groups and the surface. The oxygen atoms from two carboxylic groups $\left(\mathrm{O}_{\mathrm{COOH}}\right)$ bind to titanium atoms $\left(\mathrm{Ti}_{\text {surf }}\right)$, with a bond distance of $2.1 \AA$. The hydrogen atoms from these two groups
$\left(\mathrm{H}_{\mathrm{COOH}}\right)$ bind to bridging oxygen atoms $\left(\mathrm{O}_{\text {bridge }}\right)$ with bond distances of $1.4 \AA$.

The results for $E_{\text {int }}$ show that there is a considerable electronic interaction of $4 \mathrm{eV}$ for configuration 1 . This interaction can only take place because the TMA and the surface are deformed into the bonding geometry, which costs deformation energy. Thus, the computed adsorption energy $E_{\mathrm{b}, \mathrm{r}}$ is significantly smaller, with the difference between $E_{\text {int }}$ and $E_{\mathrm{b}, \mathrm{r}}$ being given by the sum of deformation energies of the TMA and the surface. For configuration 1 we obtain a deformation energy of $1.19(0.64) \mathrm{eV}$ for TMA (for the surface). The TMA deformation energies for the configurations 2-5 are between 0.46 and $0.79 \mathrm{eV}$. The surface deformations are in the range of $0.16-0.55 \mathrm{eV}$. The adsorption of molecules on $\mathrm{TiO}_{2}(110)$ is significantly influenced by surface defects [31]. In particular, oxygen vacancies reacting with water molecules lead to hydroxyl defects in the surface oxygen rows [47]. In order to investigate their influence on the adsorption of TMA, we included configurations with a hydroxyl defect on the surface. A hydroxyl defect is generated by adding a neutral hydrogen atom to one of the oxygen-bridge sites. This additional hydrogen atom has a dramatic effect on all five adsorption configurations shown in figure 5. Also, this influence depends on the location of the molecule relative to the hydroxyl defect. By combining the five realistic configurations of TMA on the defect-free surface with possible locations of hydroxyl underneath the molecule and after again relaxing the structure, we arrive at 19 adsorption configurations shown in figure 6. Again, the benzene ring of configuration 6 adsorbs in a nearly flat-lying configuration with a tilt angle of $8^{\circ}$. The tilt angles for configurations $7-24$ are between $1^{\circ}$ and $17^{\circ}$. 


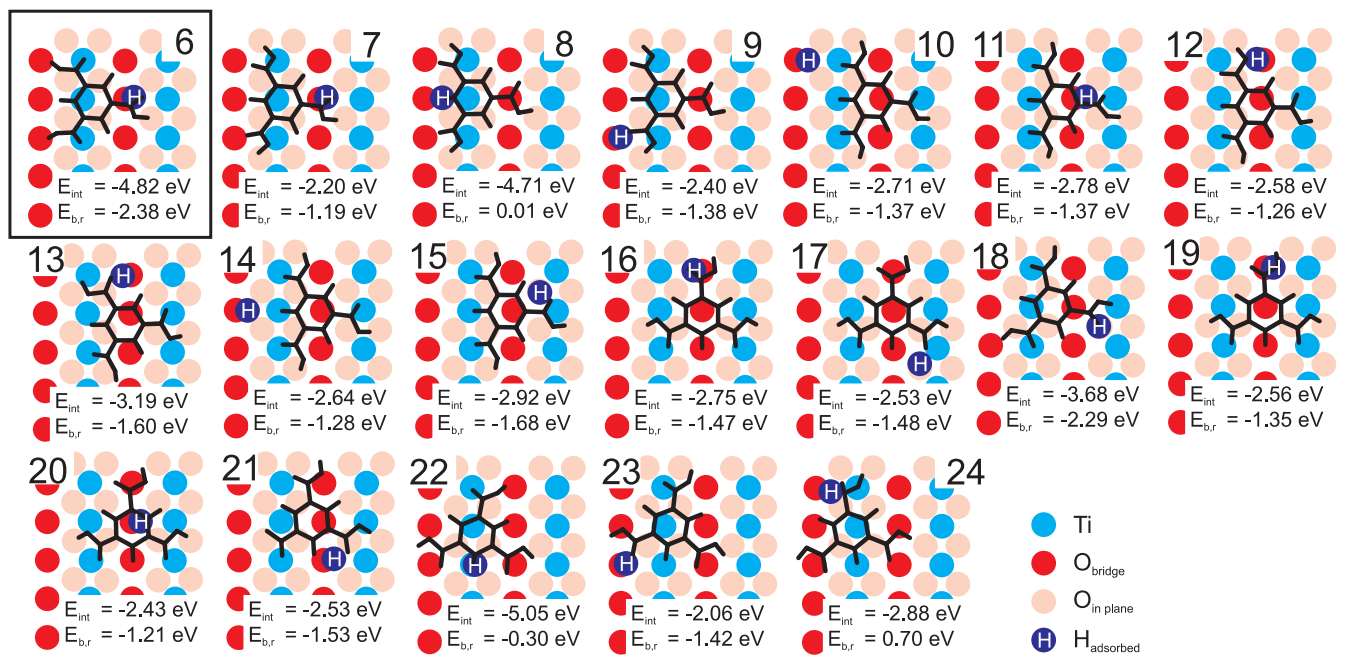

Figure 6. Relaxed configurations of the TMA molecule on the non-stoichiometric $\mathrm{TiO}_{2}(110)$ surface exhibiting a hydroxyl defect (i.e. an additional hydrogen atom attached to one of the bridging oxygen atoms). In each panel the hydroxyl defect is marked in blue.
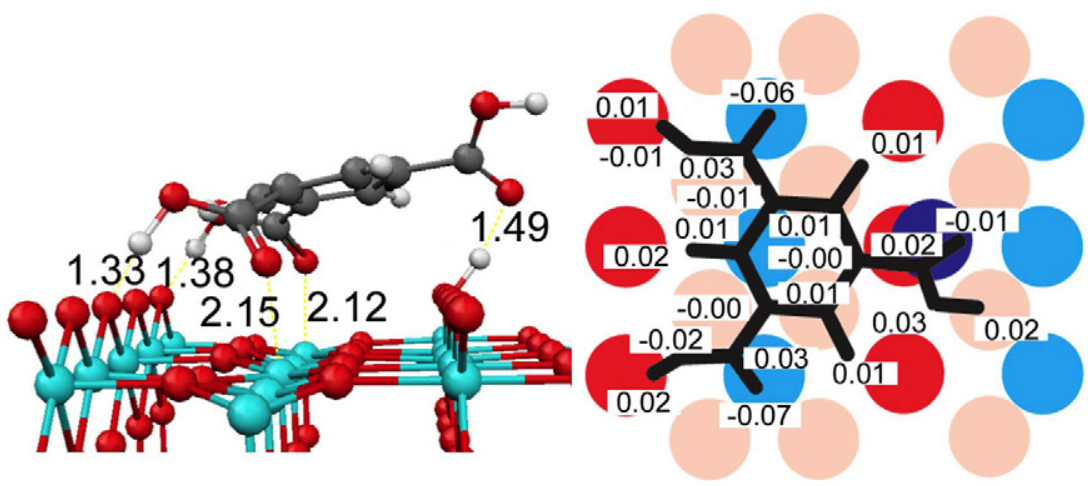

Figure 7. Most stable configuration (6). Perspective side view (left) and orthogonal projection onto the (110) plane (right). The numbers in the right panel denote the valence charge changes $\Delta Q_{\mathrm{A}}$ (in units of elementary charge $e$ ) of the TMA atoms due to the bonding to the surface. A negative value of $\Delta Q_{\mathrm{A}}$ means that the corresponding atom gains electrons with respect to the isolated molecule.

The energetically most favorable structure we found is configuration 6 . This configuration is very similar to configuration 1 of figure 5 , i.e. the most favorable configuration on the stoichiometric surface. Both in terms of $E_{\text {int }}$ and in terms of $E_{\mathrm{b}, \mathrm{r}}$, the adsorption is further stabilized by the hydroxyl defect. The reason for this stabilization is given by the carboxylic group on the right-hand side of configuration 1 or 6 (best seen in figure 7, which shows configuration 6 as a side view). On the stoichiometric surface this carboxylic group remained unbound, with a height of $3.6 \AA$ above the surface. In the case of the hydroxyl-defected surface, on the other hand, the free oxygen atom of the molecule can form a hydrogen bond with the hydrogen atom of the defect. This is reflected in the increase of $E_{\text {int }}$ by $0.83 \mathrm{eV}$ from configuration 1 to configuration 6 . To accomplish this, however, the carboxylic group has to bend in an unfavorable direction, i.e. it moves towards the surface by $0.4 \AA$ compared to configuration 1 . The corresponding increase of the molecular deformation energy by $0.4 \mathrm{eV}$, however, is overcompensated by the hydrogen bond formation, leading to an increased adsorption energy. Similarly to the data shown in figure 5, both the surface and the TMA molecule have to deform in order to enable a strong electronic interaction $E_{\text {int }}$. In configuration 6 the TMA and the surface have a deformation energy of $1.64 \mathrm{eV}$ and $0.81 \mathrm{eV}$, respectively. For the configurations 7-24 we observe TMA deformation energies in the range from $0.31 \mathrm{eV}$ (configuration 23) up to $1.87 \mathrm{eV}$ (configuration 8). The surface deformation energies go from $0.33 \mathrm{eV}$ (configuration 7) to 3.09 (configuration 22), respectively.

In order to better understand the adsorption mechanism of configuration 6 we analyze the Voronoi ${ }^{3}$ deformation density (VDD), explained in detail in [48]. Similarly to the wellknown Mulliken analysis, the VDD analysis attributes charges to atoms. This approach often yields more stable results than the Mulliken analysis, which is known to suffer from severe basis-set dependence in some cases [48]. We computed the charge deformation due to bonding between the surface and the TMA molecule as follows:

$$
\Delta Q_{\mathrm{A}}=\int_{\text {Voronoi cell A }}\left[\rho_{\text {all }}(\vec{r})-\rho_{\mathrm{TMA}}(\vec{r})-\rho_{\text {surface }}(\vec{r})\right] \mathrm{d} \vec{r} .
$$

3 For a given number of points, a Voronoi around point $\mathrm{A}$ is the region of space, which is closer to A then to any other point. 
Table 1. Voronoi charges $Q_{\mathrm{A}}$ of the most important atoms involved in TMA-surface bonding for the most stable configuration 6 on the hydroxyl-defected surface. Neutral $\mathrm{H}(\mathrm{Ti}, \mathrm{O})$ atoms have $1(4,6)$ valence electrons, respectively. Refer to figure 1 for naming.

\begin{tabular}{|c|c|c|c|}
\hline & Bond length $(\AA)$ & Charge H $(e)$ & Charge $\mathrm{O}(e)$ \\
\hline $\begin{array}{l}\mathrm{H}_{\mathrm{COOH} 1}-\mathrm{O}_{\text {bridge } 1} \\
\mathrm{H}_{\mathrm{COOH} 2}-\mathrm{O}_{\text {bridge } 2} \\
\mathrm{H}_{\text {defect }}-\mathrm{O}_{\mathrm{COOH}}\end{array}$ & $\begin{array}{l}1.33 \\
1.38 \\
1.49\end{array}$ & $\begin{array}{l}-0.88 \\
-0.87 \\
-0.87\end{array}$ & $\begin{array}{l}-6.32 \\
-6.33 \\
-6.21\end{array}$ \\
\hline & Bond length $(\AA)$ & Charge Ti $(e)$ & Charge $\mathrm{O}(e)$ \\
\hline $\begin{array}{l}\mathrm{Ti}_{\text {surf } 1}-\mathrm{O}_{\mathrm{COOH} 1} \\
\mathrm{Ti}_{\text {surf } 2}-\mathrm{O}_{\mathrm{COOH} 2} \\
\mathrm{Ti}_{\text {bulk } 1}-\mathrm{O}_{\text {bulk1 }} \\
\mathrm{Ti}_{\text {bulk2 } 2}-\mathrm{O}_{\text {bulk2 }}\end{array}$ & $\begin{array}{l}2.12 \\
2.15 \\
1.94 \\
1.98\end{array}$ & $\begin{array}{l}-3.29 \\
-3.29 \\
-3.30 \\
-3.30\end{array}$ & $\begin{array}{l}-6.23 \\
-6.22 \\
-6.35 \\
-6.35\end{array}$ \\
\hline
\end{tabular}

The bonding of the molecule towards the surface is mainly caused by the bonds between the titanium rows and the oxygen atoms of two carboxylic groups $\left(\mathrm{Ti}_{\text {surf }}-\mathrm{O}_{\mathrm{COOH}}\right.$ bonds, caused by electrostatic interaction) and between the bridging oxygen row and the hydrogen atoms of the same carboxylic groups ( $\mathrm{O}_{\text {bridge }}-\mathrm{H}_{\mathrm{COOH}}$ hydrogen-bridge bonds). Additionally, the oxygen atom of the third carboxylic group binds towards the hydroxyl defect $\left(\mathrm{O}_{\mathrm{COOH}}-\mathrm{H}_{\text {defect }}\right.$ hydrogen-bridge bond $)$.

Our calculations reveal only a small charge transfer from the surface to the molecule upon bond formation. However, the residual molecule back-donates electrons towards the surface. Thus, in sum the entire molecule loses $\sim 0.03$ electrons to the surface. The valence charge changes $\Delta Q_{\mathrm{A}}$ for the Voronoi cells of the individual atoms are given in figure 7 , whereas table 1 lists the Voronoi charges $Q_{\mathrm{A}}$ for the atoms involved in the TMA-surface bond formation.

The $\mathrm{Ti}_{\text {surf }}-\mathrm{O}_{\mathrm{COOH}}$ bonds have a length of 2.15/2.12 $\AA$, which is slightly larger than the Ti-O bond lengths occurring in bulk $\mathrm{TiO}_{2}$ (1.94 and $1.98 \AA$ in our calculation framework). The $\mathrm{O}_{\mathrm{COOH}}$ atoms gain $0.06 / 0.07$ electrons, while the $\mathrm{Ti}_{\text {surf }}$ atoms lose 0.04/0.04 electrons. The bond length and the charge distribution (compared to the bulk values, see table 1) reflect that the binding between the molecular oxygen and the surface titanium is not as strong as the Ti-O bonds within the bulk.

The $\mathrm{O}_{\text {bridge }}-\mathrm{H}_{\mathrm{COOH}}$ bonds have a length of $1.33 / 1.38 \AA$. The $\mathrm{O}_{\text {bridge }}$ atoms gain $0.04 / 0.04$ electrons, while the $\mathrm{H}_{\mathrm{COOH}}$ atoms lose 0.02/0.01 electrons. Finally, the $\mathrm{O}_{\mathrm{COOH}}-\mathrm{H}_{\text {defect }}$ bond has a length of $1.49 \AA$. Here, the charge redistributes as follows: the $\mathrm{O}_{\mathrm{COOH}}$ gains 0.01 electrons, whereas there is virtually no change at the $\mathrm{H}_{\text {defect }}$.

Besides configurations where the TMA molecule lies flat on the surface, we also tested configurations with uprightstanding molecules on the stoichiometric surface. The most stable configuration is shown in figure 8. Here the TMA molecule is almost planar, with only a slight deformation of the $\mathrm{COOH}$ groups binding to the surface.

We get an adsorption energy of $-1.45 \mathrm{eV}$, with the TMA having a deformation energy of $0.46 \mathrm{eV}$. The absolute value of the adsorption energy is higher (more stable) than for the cases of non-tilted upright-standing molecules. However, the best configurations for flat-lying molecules are still significantly stronger bound. From our results we conclude that TMA on

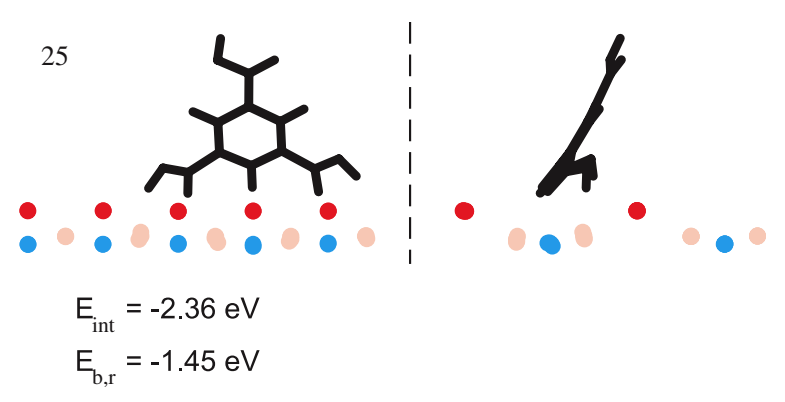

Figure 8. The most stable configuration for a TMA molecule standing upright on the clean $\mathrm{TiO}_{2}$ surface, shown as a projection onto the (110) plane (left) and the (001) plane (right).

$\mathrm{TiO}_{2}$ (110) always adsorbs in a flat-lying configuration, and upright-standing molecules do not occur.

Finally, we also address the issue of TMA deprotonation. It is well known that carboxylic acids adsorbed onto metal surfaces such as $\mathrm{Cu}(110)$ undergo deprotonation and adsorb as carboxylate $[49,50]$. Also on $\mathrm{TiO}_{2}(110)$, deprotonation of the carboxylic group has been suggested by various groups [51, 52]. Consequently, we have also tested several geometries with a deprotonated TMA molecule. To this end we move the hydrogen atom of one carboxylic group to an oxygen-bridge site far away, thus forming a hydroxyl defect. Note that at electronic self-consistency, DFT yields that the deprotonated TMA molecule is again charge-neutral, i.e. the hydrogen atom is displaced as $\mathrm{H}^{0}$ rather than as $\mathrm{H}^{+}$. The most stable relaxed structures for these configurations are shown in figure 9. For these configurations, the evaluation of $E_{1}$ (i.e. the TMA molecule without the surface, but kept in the geometry in which it adsorbs on the surface) is not possible in a useful way since it involves the deprotonation, i.e. the displacement of the hydrogen atom by several ångströms. The associated 'deformation energy', i.e. the difference to $E_{3}$, would amount to several electronvolts. Consequently, the resulting binding energy $E_{\text {int }}$ also yields no useful results in this case. The underlying concept, i.e. separating the adsorption components (molecule and surface) at unchanged internal structure, is not useful for cases in which a chemical reaction has changed the attribution of atoms to the two components. Therefore we do not discuss the deformation energies and $E_{\text {int }}$ for the deprotonation-assisted adsorption of TMA, but restrict ourselves to the discussion of $E_{\mathrm{b}, \mathrm{r}}$, which still has a welldefined physical significance.

The most favorable deprotonated structure is configuration 26 , with a binding energy of $E_{\mathrm{b}, \mathrm{r}}=-2.01 \mathrm{eV}$. Thus, based on these theoretical results, we conclude that deprotonation for TMA is less favorable by $0.37 \mathrm{eV}$ for adsorption on the hydroxylated surface and less favorable by $0.15 \mathrm{eV}$ on the clean $\mathrm{TiO}_{2}(110)$ surface. This is descriptively explainable, as the third carboxylic group is hindered from forming a hydrogen bond to a hydroxyl group as calculated in configuration 6 . Contrary, as shown in configuration 26 , the carboxylate group bends toward the neighboring $\mathrm{Ti}_{\text {surf }}$ row, inducing a large strain in both molecule and surface.

Table 2 summarizes the adsorption and deformation energies for the most favorable structures (configurations 1, 6 

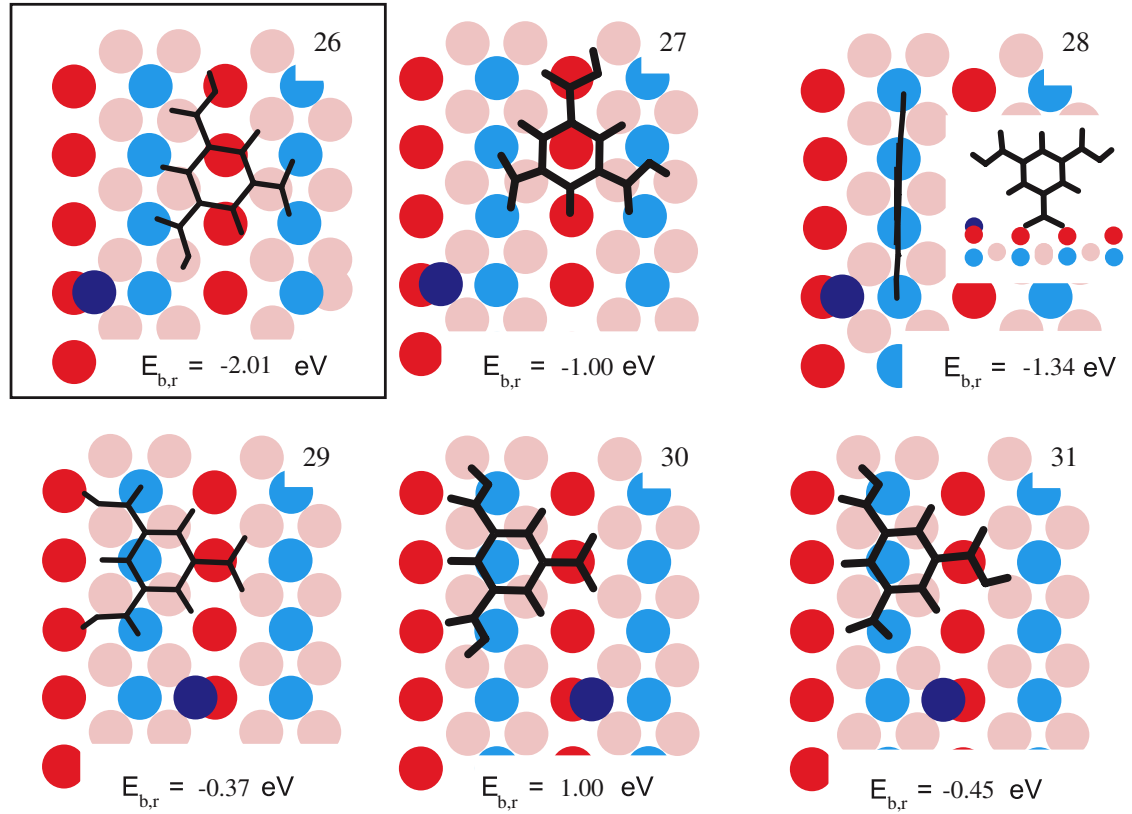

Figure 9. The six most realistic relaxed configurations of a deprotonated TMA molecule on the stoichiometric $\mathrm{TiO}_{2}(110)$ surface. Configuration 26 yields the most stable adsorption geometry.
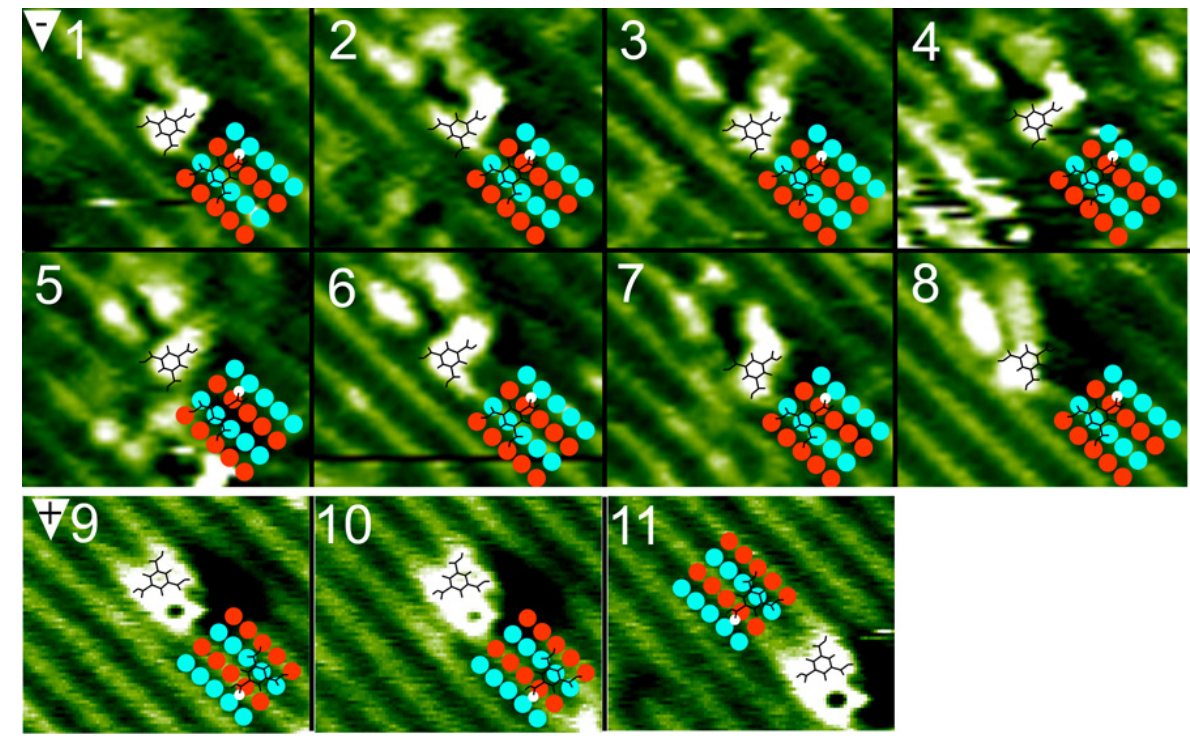

Figure 10. Comparison of the best adsorption geometry obtained from DFT with NC-AFM results. (1)-(8) show images of the corresponding molecules extracted from figure 2 taken in the protrusion mode. (9)-(11) present images taken in the hole mode [33], which has been explained by a positively terminated tip (full scan not shown). The molecules are immobile at room temperature, and diffusion is only observed extremely rarely. From the numbered molecules presented in figure 2, no. (8) is the only molecule disappearing within a time frame of $\sim 75 \mathrm{~min}$.

and 26) on the clean and hydroxylated $\mathrm{TiO}_{2}$ surface, including a deprotonated molecule. The data clearly show that chemical interaction is accompanied by significant elastic deformation of both the molecule and the substrate. While this could be expected for a large, flat (and thus flexible) organic molecule, a deformation energy of up to $1 \mathrm{eV}$ and more of an anorganic oxide surface represents a surprising result.

Furthermore, our data indicate that the additional binding energy due to the hydroxyl defect constitutes a barrier that would have to be overcome to move the molecule from a hydroxyl defect to a defect-free region of the surface. In effect, TMA molecules on $\mathrm{TiO}_{2}$ (110) might be trapped at such defects, and diffusion along the surface would be suppressed. This conjecture is supported by our experimental observation that TMA is immobile at room temperature.

In order to compare the most favorable DFT geometry with our experimental findings, we superimposed the favorable DFT geometry with experimental data (figure 10). We compare the experiment and the most favorable structure from theory for two different contrast modes. Images 1-8 of figure 10 are taken 
Table 2. Energies of the most stable configurations on the clean $\mathrm{TiO}_{2}$ surface, on the hydroxyl-defected surface, and for a TMA molecule that deprotonates on the clean $\mathrm{TiO}_{2}$ surface, creating a hydroxyl defect (all systems are charge-neutral). The deformation energies of the $\mathrm{TiO}_{2}$ surface and of the TMA molecule are given by $E_{\text {deform }}\left(\mathrm{TiO}_{2}\right)=E_{2}-E_{4}$ and by $E_{\text {deform }}(\mathrm{TMA})=E_{1}-E_{3}$, respectively.

\begin{tabular}{llll}
\hline & $\begin{array}{l}\text { TMA on } \\
\text { clean } \mathrm{TiO}_{2} \\
\text { (config. 1) }\end{array}$ & $\begin{array}{l}\mathrm{TMA} \text { on } \mathrm{TiO}_{2} \\
\text { with OH } \\
\text { (config. 6) }\end{array}$ & $\begin{array}{l}\text { Depr. TMA } \\
\text { on } \mathrm{TiO}_{2} \\
\text { (config. 26) }\end{array}$ \\
\hline$E_{\text {int }}(\mathrm{eV})$ & -3.99 & -4.82 & \\
$E_{\mathrm{b}, \mathrm{r}}(\mathrm{eV})$ & -2.16 & -2.38 & -2.01 \\
$E_{\text {deform }}\left(\mathrm{TiO}_{2}\right)(\mathrm{eV})$ & 0.64 & 0.81 & \\
$E_{\text {deform }}(\mathrm{TMA})(\mathrm{eV})$ & 1.19 & 1.63 & \\
\hline
\end{tabular}

from figure 2, which was assigned to protrusion mode. Images 9-11 in figure 10, on the other hand, are taken in the so-called hole mode, which has been explained by a positive tip. We can identify the position relative to the $[\overline{1} 10]$ direction and find that the position of the experimentally observed feature agrees with the optimum geometry from theory, which is shown in figure 7. In both contrast modes, the orientation of the TMA molecule relative to the surface is, as expected, the same.

\section{Conclusion}

We have presented a combined NC-AFM and DFT study on the adsorption behavior of $\mathrm{TMA}$ on $\mathrm{TiO}_{2}(110)$ at room temperature, focusing on the adsorption geometry at low coverages.

The NC-AFM images reveal stable molecular structures centered on top of the in-plane titanium rows. Our experimental results suggest that the molecules anchor to special surface sites, which most likely are hydroxyl defects, as supported by our DFT calculations.

The optimum adsorption geometry as obtained by our theoretical calculations is a flat-lying molecule. Deprotonation of the carboxylic groups is not favored based on our DFT results. The position along the [ $\overline{1} 10]$ direction as revealed by NC-AFM is confirmed. Binding mostly results from two of the three carboxylic groups, which interact with the surface oxygen rows and the adjacent titanium rows. Furthermore, the molecule is more strongly bound to the surface when a hydroxyl defect is present, due to an additional bond involving the third carboxylic group. This constitutes a barrier for removing the molecule from the defect, which might explain why the molecule is rather immobile at room temperature.

\section{Acknowledgments}

This work has been supported by the German Research Foundation (DFG) through the Emmy Noether programme (KU1980/1-2) and by research grant RO1318/6-1.

\section{References}

[1] Ertl G and Freund H-J 1999 Phys. Today 5232

[2] Felhösi I, Kálmán E and Póczik P 2002 Russ. J. Electrochem. 38230
[3] Chaki N K and Vijayamohanan K 2002 Biosens. Bioelectron. 171

[4] Whitesides G M, Mathias J P and Seto C T 1991 Science 2541312

[5] Service R 2004 Science 3031136

[6] Rosei F, Schunack M, Naitoh Y, Jiang P, Gourdon A, Lægsgaard E, Stensgaard I, Joachim C and Besenbacher F 2003 Prog. Surf. Sci. 7195

[7] Tautz F S 2007 Prog. Surf. Sci. 82479

[8] Rohrbach A, Hafner J and Kresse G 2004 Phys. Rev. B 69075413

[9] Hammer B and Nørskov J K 2000 Adv. Catal. 4571

[10] Diebold U 2003 Surf. Sci. Rep. 4853

[11] Watkins M, Trevethan T, Sushko M L and Shluger A L 2008 J. Phys. Chem. C 1124226

[12] Trevethan T and Shluger A L 2008 J. Phys. Chem. C 11219577

[13] Nyberg M, Hasselström J, Karis O, Wassdahl N, Weinelt M, Nilsson A and Pettersson L G M 2000 J. Chem. Phys. 1125420

[14] Di Felice R, Selloni A and Molinari E 2002 J. Phys. Chem. 1071151

[15] Fukui K-I, Onishi H and Iwasawa Y 1997 Chem. Phys. Lett. 280296

[16] Sasahara A, Uetsuka H, Ishibashi T A and Onishi H 2002 Appl. Surf. Sci. 188265

[17] Tekiel A, Prauzner-Bechcicki J S, Godlewski S, Budzioch J and Szymonski M 2008 J. Phys. Chem. C 11212606

[18] Rahe P, Nimmrich M, Nefedov A, Naboka M, Wöll C and Kühnle A 2009 J. Phys. Chem. C 11317471

[19] Dmitriev A, Lin N, Weckesser J, Barth J V and Kern K 2002 J. Phys. Chem. B 1066907

[20] Ito E, Konno K, Noh J, Kanai K, Ouchi Y, Seki K and Hara M 2005 Appl. Surf. Sci. 244584

[21] Ye Y C, Sun W, Wang Y F, Shao X, Xu X G, Cheng F, Li J L and Wu K 2007 J. Phys. Chem. C 11110138

[22] Sheerin G and Cafolla A A 2005 Surf. Sci. 577211

[23] MacLeod J M, Ivasenko O, Perepichka D F and Rosei F 2007 Nanotechnology 18424031

[24] Lackinger M, Griessl S, Heckl W A, Hietschold M and Flynn G W 2005 Langmuir 214984

[25] Griessl S, Lackinger M, Edelwirth M, Hietschold M and Heckl W M 2002 Single Mol. 325

[26] Bates S P, Kresse G and Gillan M J 1998 Surf. Sci. 409336

[27] McGill P R and Idriss H 2008 Surf. Sci. 6023688

[28] Guo Q and Williams E M 1999 Surf. Sci. 433322

[29] Ordejón P, Artacho E and Soler J M 1996 Phys. Rev. B 53 R 10441

[30] Soler J M, Artacho E, Gale J D, Garcia A, Junquera J, Ordejon P and Sanchez-Portal D 2002 J. Phys.: Condens. Matter 142745

[31] Schütte J, Bechstein R, Rahe P, Rohlfing M, Kühnle A and Langhals H 2009 Phys. Rev. B 79045428

[32] Rahe P, Bechstein R, Schütte J, Ostendorf F and Kühnle A 2008 Phys. Rev. B 77195410

[33] Lauritsen J V, Foster A S, Olesen G H, Christensen M C, Kühnle A, Helveg S, Rostrup-Nielsen J R, Clausen B S, Reichling M and Besenbacher F 2006 Nanotechnology 173436

[34] Enevoldsen G H, Foster A S, Christensen M C, Lauritsen J V and Besenbacher F 2007 Phys. Rev. B 76205415

[35] Bechstein R, González C, Schütte J, Jelínek P, Pérez R and Kühnle A 2009 Nanotechnology 20505703

[36] Yurtsever A, Sugimoto Y, Abe M and Morita S 2010 Nanotechnology 21165702

[37] Ceperley D M and Alder B J 1980 Phys. Rev. Lett. 45566

[38] Marini A, García-González P and Rubio A 2006 Phys. Rev. Lett. 96136404

[39] Harl J, Schimka L and Kresse G 2010 Phys. Rev. B 81115126

[40] Rohlfing M and Bredow T 2008 Phys. Rev. Lett. 101266106 
[41] Troullier N and Martins J L 1991 Phys. Rev. B 431993

[42] Cromer D T and Herrington K 1955 J. Am. Chem. Soc. 774708

[43] Ostlund N S and Merrifield D L 1976 Chem. Phys. Lett. 39612

[44] Boys S F and Bernardi F 1970 Mol. Phys. 19553

[45] Broyden C G 1970 J. Appl. Math. 676

[46] Hestenes M R and Stiefel E 1952 J. Res. Natl Bur. Stand. 49 409

[47] Wendt S et al 2005 Surf. Sci. 598226

[48] Guerra C F, Handgraaf J, Baerends E J and Bickelhaupt F M 2004 J. Comput. Chem. 25189
[49] Frederick B, Ashton M, Richardson N and Jones T 1993 Surf. Sci. 29233

[50] Surman M, Lackey D and King D A 1986 J. Electron Spectrosc. Relat. Phenom. 39245

[51] Thomas A, Flavell W, Chatwin C, Kumarasinghe A, Rayner S, Kirkham P, Tsoutsou D, Johal T and Patel S 2007 Surf. Sci. 6013828

[52] Schnadt J, O'Shea J N, Patthey L, Schiessling J, Krempaský J, Shi M, Mårtensson N and Brühwiler P A 2003 Surf. Sci. 54474 